\section{FILOSOFÍA Y DERECHOS HUMANOS}

\author{
Ricardo Parellada \\ (Universidad Complutense)
}

\section{PHILOSOPHY AND HUMAN RIGHTS}

RESUMEN: Se examina la relevancia de los análisis filosóficos para la conceptualización y para la realización de los derechos humanos, a partir de dos episodios concretos: la elaboración de la declaración universal de los derechos humanos y los planteamientos recientes sobre la justicia global. Se propone que la fuerza y la autoridad moral de los derechos humanos no depende de que se haya llevado a cabo un examen exhaustivo de esta noción, pero también que esa autoridad no es compatible con cualquier teoria sobre ello. Y se defiende la relevancia del análisis filosófico de la idea de justicia global y la irrelevancia de la filosofía para su realización.

PALABRAS CLAVE: Derechos humanos; análisis filosófico; Naciones Unidas; justicia global.

En segundo lugar, me propongo contemplar también los planteamientos filosóficos desde las expectativas y las objeciones de otras disciplinas. En muchos ámbitos confluyen análisis filosóficos o conceptuales con los de otras disciplinas, y el entendimiento muchas veces no es fácil. En la filosofía de la ciencia, la filosofía de la mente o la filosofía de la acción, por ejemplo, se producen encuentros y desencuentros entre los análisis filosóficos y las propuestas de las ciencias, la psicología o el derecho. Desde muchas disciplinas se contempla con gran escepticismo que la filosofía pueda ofrecer algo que no forme parte del trabajo explícito de estas disciplinas. Los filósofos tienen grandes dificultades tanto a la hora de examinar sus problemas como a la hora de mostrar a los demás su complejidad y sus implicaciones. Esta dificultad se da también con los derechos humanos. Al examinar los análisis filosóficos sobre los derechos humanos, y su relevancia para la reflexión y la acción, me preguntaré también por la visión que transmiten otras disciplinas de estos análisis. Aunque yo no creo que exista una manera general de plantear la relación entre la filosofía y otras disciplinas, me parece que el caso de los derechos humanos es muy ilustrativo para reflexionar sobre esta relación.

Para abordar ambas cuestiones -la especificidad del enfoque filosófico y la relación entre la filosofía y otras consideración la perspectiva teórica y conceptual. 
disciplinas, a propósito de los derechos humanos- me voy a centrar en dos episodios de gran trascendencia en el último siglo: por un lado, la elaboración de la Declaración Universal de los Derechos Humanos, proclamada a finales de 1948, y, por otro lado, la apuesta por planteamientos globales sobre la justicia y los derechos humanos, que se han generalizado en la primera década del siglo XXI. La elaboración de la declaración y el discurso sobre la justicia global pretenden servir tanto a la inteligencia como a la realización de los derechos humanos, y en ambas empresas confluyen y cooperan perspectivas disciplinares e intereses prácticos diversos. Al tratarse de fenómenos tan significativos, espero que su examen permita dar concreción a la discusión y posibilitar a la vez una perspectiva más amplia sobre la cuestión general acerca de la relevancia de la filosofía para los derechos humanos.

\section{LA FILOSOFÍA Y LA DECLARACIÓN DE LOS DERECHOS HUMANOS}

Los derechos humanos tienen una larga historia antes de la declaración de 1948'. Mas esta declaración tiene una importancia singular porque reúne y depura elementos anteriores, se presenta con vocación universal, fue proclamada por la Asamblea General de unas incipientes Naciones Unidas, sobre las ruinas aún humeantes de la Segunda Guerra Mundial, y constituye, a partir de entonces, la referencia indiscutible tanto del discurso, los convenios y los documentos de derechos humanos, como de la mayoría de las constituciones democráticas posteriores, incluyendo las de los paises perdedores de la guerra, que fueron excluidos al principio de las Naciones Unidas. Durante la elaboración de la declaración tuvieron lugar infinidad de discusiones y negociaciones. A mi modo de ver, estas complejas discusiones para consensuar la declaración son muy ilustrativas sobre el papel de las distintas perspectivas teóricas e intereses prácticos en la construcción y la formulación moderna de los derechos humanos.

La declaración universal fue elaborada por una comisión delegada del Comité de Asuntos Sociales y Humanitarios o tercer comité de las Naciones Unidas². Durante los casi dos años que transcurrieron desde las primeras reuniones de la comisión a principios de 1947 hasta la proclamación de la declaración el 10 de diciembre de 1948, tuvieron lugar discusiones sobre cada palabra y cada expresión, primero en el seno de la comisión y después en reuniones plenarias del tercer comité. La tarea más importante fue la de la comisión, que presentó un documento muy elaborado al tercer comité, que a su vez sometió a discusión y votación cada uno de sus párrafos antes de presentarlo a la asamblea. La comisión estaba formada por dieciocho miembros, los vencedores de la Segunda Guerra Mundial (EEUU, Reino Unido, Francia, China y URSS) y otros trece representantes que fueron rotando entre el resto de los miembros de la ONU. La comisión estaba presidida por Eleanor Roosevelt, viuda del presidente de EEUU, el vicepresidente era el diplomático chino Peng-chun Chang y el secretario el profesor libanés Charles Malik. Estos dos últimos fueron quienes desempeñaron un papel más activo en las discusiones filosóficas de la comisión.

La noción de derechos humanos aparece en siete lugares clave de la Carta fundacional de las Naciones Unidas aprobada en San Francisco en la primavera de 1945. Esta noción aparecía sin ningún tipo de explicación o enumeración, y la comisión delegada del tercer comité recibió el encargo de trabajar sobre ella y elaborar una carta de los derechos más básicos que todos los países miembros pudieran aceptar. Pero este encargo era vago y fue la propia comisión, tras sus primeras reuniones, la que tomó la decisión de dejar de lado la explicitación de un régimen sancionador para los incumplimientos de los principios acordados. Un régimen sancionador, como el asociado a toda norma jurídica, demandaría instituciones y mecanismos internacionales de control imposibles de vislumbrar, prever o consensuar en aquellos momentos, ni siquiera en términos completamente generales. De ahí que la comisión decidiera elaborar una declaración de principios que pudiese alcanzar una autoridad moral internacional, inspirar las constituciones nuevas y las leyes más generales de los estados y servir para denunciar las prácticas más injustas.

La comisión encargó la elaboración de un primer borrador a John Humphrey, director canadiense de la División de Derechos Humanos del Secretariado de la ONU. Humphrey y su equipo llevaron a cabo una labor exhaustiva de recopilación de documentos de derechos de los individuos y principios de la vida social en diversas naciones y culturas, y presentaron a la comisión un documento muy heterogéneo, acompañado de más de cuatrocientas páginas de comentarios. René Cassin, prestigioso jurista francés de 
origen judio, que habia perdido a veinte familiares en los campos de concentración nazis, habia sido el principal asesor jurídico del general Charles de Gaulle en el exilio y presidia entonces el Consejo de Estado francés, fue encargado de sintetizar y dar forma a los ricos materiales aportados por Humphrey. El borrador de Cassin, con su división en un preámbulo y una enumeración de principios, fue la base de las discusiones de la comisión, pero ésta sometió todos sus puntos a discusiones exhaustivas, tomó las decisiones más controvertidas y les dio la ordenación y la formulación final, por lo que no es correcto atribuir una autoría singular de la declaración a Cassin, como tampoco a Humphrey. La declaración fue fruto de una labor colegiada, paciente y minuciosa, durante la cual, como he señalado más arriba, el representante libanés, Charles Malik, y el representante chino, Peng-chun Chang, ofrecieron las reflexiones más profundas.

Charles Malik era un profesor de filosofía libanés, de familia cristiana pero con antecedentes familiares procedentes de las tres grandes religiones de Oriente Medio, que habia pasado parte de sus años de formación junto a Whitehead en EEUU y junto a Heidegger en Alemania, y cuya incorporación a la delegación libanesa ante la ONU fue su primera experiencia diplomática. Por su parte, Peng-chun Chang era un experimentado embajador, jurista de profesión y filósofo de vocación, que poseía profundos conocimientos tanto de tradiciones orientales (en particular la cultura china), como occidentales. Durante la discusión de muchas normas y principios de respeto a las personas, Chang aportaba y comentaba referencias a antecedentes de esas ideas en diversas culturas.

Pues bien, desde un principio Malik proclamó con solemnidad la vocación filosófica y la trascendencia intelectual de la declaración de derechos humanos: "Cuando hablamos de derechos humanos estamos planteando la pregunta fundamental, ¿qué es el hombre?". "Al disentir sobre los derechos humanos, en realidad disentimos -aseguraba- sobre la naturaleza de la persona. ¿Es el hombre meramente un ser social? ¿Es meramente un animal? ¿Es simplemente un agente económico?". Malik consideraba que el mayor peligro de la época lo planteaba el colectivismo, que demandaba "la extinción de la persona humana como tal en su propia individualidad e inviolabilidad última", y propuso cuatro principios orientadores: (1) la persona humana es más importante que cualquier grupo nacional o cultural al que pertenezca; (2) la mente y la conciencia humanas son su posesión más sagrada e inviolable; (3) toda presión del estado, la iglesia u otro grupo para forzar el asentimiento es inaceptable; (4) dado que los grupos, como los individuos, pueden estar en lo cierto o equivocados, la libertad de conciencia del individuo debe ser suprema.

La comisión de derechos humanos asumió esta propuesta, pero convino también, desde un principio, que se subrayaría por igual el carácter social del ser humano, asumiendo el reto de ofrecer una noción del ser humano anclada en su autonomía individual y en su carácter social y rechazando la unilateralidad del individualismo liberal y del colectivismo socialista. Fue Chang quien propuso la inclusión del carácter social del ser humano desde el primer artículo de la declaración, remitiendo para ello al término chino ren. De acuerdo con la explicación de Chang, este término denota la interdependencia de los seres humanos, la fraternidad y la implicación emocional de unos con otros. Esta idea debía acompañar a la racionalidad como los dos rasgos definitorios de la naturaleza humana. Desgraciadamente, la propuesta de Chang tuvo una deficiente plasmación lingüística, al traducirse el término ren por conscience en inglés, el idioma original de la declaración (aunque Cassin escribió su borrador en francés). El primer artículo de la declaración afirma que los seres humanos están dotados de razón y conciencia, pero el sentido original era razón y empatía o inclinación fraternal de los unos hacia los otros.

En plena discusión sobre los individuos y la sociedad, se alzó la voz impaciente de Hansa Metha, la representante de la India. Metha era una activista y llevó a cabo una labor ingente en su país, antes y después de la independencia de la India en agosto de 1947 y de la proclamación de la declaración, contra prácticas como el purdah o reclusión femenina, el matrimonio infantil, los derechos desiguales de herencia entre hombres y mujeres y la prohibición de los matrimonios entre miembros de distintas castas. Durante los últimos retoques a la declaración antes de enviarla al tercer comité, Metha consiguió que se aceptaran formulaciones neutras en el género, sustituyendo las expresiones "todos los hombres" y "ningún hombre" por "todos" y "nadie". Curiosamente este cambio, que no había prosperado en los primeros momentos, se introdujo en contra del criterio de la mujer que desempeñaba el papel más relevante en la comisión, su presidente, Eleanor Roosevelt, quien 
consideraba que la expresión inglesa "all men" se refería sin ambigüedad a todos los seres humanos.

Pero en el caso del individuo y la sociedad, Metha no consideraba necesarios tantos melindres: la comisión no debía "discutir sobre ideología: si el ser humano viene antes o después de la sociedad", sino simplemente "afirmar la fe en los derechos humanos". La respuesta del filósofo Malik no se hizo esperar: "cualquier cosa que diga, señora, tiene presupuestos ideológicos $y$, por mucho que usted quiera ignorarlos, están ahí, y se pueden esconder o traer a la luz, para verlos bien y poder criticarlos". A mi juicio, esta discusión ilustra muy bien las diferencias entre actitudes más teóricas y más prácticas hacia los derechos humanos. Hansa Metha estaba volcada a combatir prácticas injustas y contemplaba a esta luz la elaboración de la carta de derechos. Desde la conciencia y el compromiso social, las disquisiciones conceptuales pueden resultar carentes de sentido $y$, sobre todo, irrelevantes para la realización de los derechos humanos y la lucha contra la ignominia o la miseria. En mi opinión, el contraste entre la voluntad analítica de Charles Malik y la impaciencia resolutiva de Hansa Metha en la elaboración de la declaración universal de los derechos humanos sirve para ilustrar la contraposición, que se reproduce de muchas maneras, entre la parsimonia de la indagación teórica y la urgencia de la realización efectiva de los derechos humanos. Pero antes de vincular este contraste con la pregunta inicial por la relevancia de las indagaciones filosóficas, me gustaría señalar un par de elementos adicionales de la declaración.

Para salvaguardar su universalidad, la declaración no apela a los fundamentos religiosos aducidos por otras cartas anteriores de derechos (aunque Brasil propuso añadir una referencia al creador en el tercer comité). En el último instante, y a propuesta belga, se suprimió también una alusión a la naturaleza. El concepto que en momentos clave de la declaración pasa a desempeñar un papel central es el de dignidad humana: "la dignidad intrínseca y los derechos iguales de todos los miembros de la familia humana son el fundamento de la libertad, la justicia y la paz en el mundo", reza la frase inicial del preámbulo. La carta fundacional de las Naciones Unidas había afirmado la fe en la libertad y la democracia. La declaración la interpreta como fe en los derechos humanos, en la dignidad y el valor de la persona humana y en la igualdad de derechos de hombres y mujeres. Como dice Glendon, "para tratarse de un documento que evita toda inspiración divina, se trata de un montón de $\mathrm{fe}^{\text {"3. }}$. En la historia de los derechos humanos se da un tránsito del fundamento religioso a la naturaleza y de la naturaleza a la dignidad. La remisión de la dignidad a la autonomía o a la empatía abre todo el abigarrado mundo de la reflexión filosófica, que es compleja y apasionante. Pero los miembros de la comisión de derechos humanos de la ONU que elaboraron la declaración no podían pararse a especular y tuvieron que hacer un esfuerzo titánico para consensuar un texto que llegara a la asamblea general antes de que lo impidiera la guerra fría. Este texto fue aprobado por cuarenta y ocho votos a favor, ocho abstenciones y ningún voto en contra.

Los derechos humanos se pueden remitir a la divinidad, la naturaleza, la dignidad, el consenso o sus contrarios. También se puede negar que la noción de fundamentación tenga sentido. Pero para sostener lo uno o lo otro es necesario articular un discurso paciente e informado. Ésta ha sido siempre la tarea de la filosofía. Podemos preguntarnos si son necesarios, o tienen alguna utilidad, estos análisis para tener una concepción clara de los derechos humanos o para luchar por su respeto universal. Desde la lucha contra la miseria y la ignominia, es fácil contemplar la discusión conceptual como un pasatiempo fútil o incluso irresponsable. Y desde la economía, el derecho o las relaciones internacionales, se comprende bien la importancia de cualquiera de esas otras disciplinas, pero es difícil vislumbrar algún sentido en las digresiones filosóficas o concederles alguna relevancia.

Sin embargo, en los primeros años de existencia de las Naciones Unidas, la Organización para la Educación, la Ciencia y la Cultura (Unesco) sí parecía conceder relevancia a las indagaciones filosóficas y, mientras la comisión de derechos humanos preparaba la carta internacional, consultó a eminentes pensadores y personalidades que podrían ayudar en la labor más teórica de la comisión. La Unesco pidió a estas personalidades que enviasen sus reflexiones sobre la siguiente cuestión: "¿es posible que desde culturas y tradiciones muy diversas se pueda llegar a un acuerdo acerca de una carta internacional de derechos de los individuos que todas las naciones deberán respetar?". Algunas de las personalidades consultadas fueron Aldous Huxley, Pierre Teilhard de Chardin, Mahatma Gandhi, Benedetto Croce y Salvador de Madariaga. Sus respuestas se reunieron a lo largo de 1947 y 1948, pero no sirvieron para arro- 
jar gran luz sobre el problema y no fueron aprovechadas por la comisión de derechos humanos. Entre la disparidad de respuestas, han tenido especial eco las consideraciones del filósofo francés Jacques Maritain en la introducción a la obra colectiva en la que se publicaron ${ }^{4}$.

Maritain sostiene que culturas y concepciones intelectuales y religiosas diversas pueden llegar a un acuerdo sobre los principios más básicos del respeto a los individuos en el orden social, porque la finalidad del acuerdo es práctica. A decir de Maritain, no se trata de llegar a un acuerdo acerca de la noción de persona, su valor intrínseco o las razones para respetar su conciencia como el reducto más sagrado de su personalidad, sino de formular principios claros que recojan los derechos básicos, civiles y políticos y sociales, económicos y culturales, para poder hablar de vida digna y libre desarrollo de la personalidad. A su juicio, los pensadores religiosos y racionalistas no podrán nunca ponerse de acuerdo sobre las razones para atribuir dignidad y derechos básicos a las personas, pero sí podrán elaborar las normas y principios de conducta recogidos en la declaración de derechos. Por debajo de los razonamientos explícitos resulta efectivo, a juicio de Maritain, un fondo intelectual que corresponde a la época y permite el acuerdo práctico a pesar de profundas discrepancias teóricas. La propuesta de Maritain ha tenido singular influencia en la reflexión sobre los derechos humanos, y su actualización más conocida es quizá la reelaboración llevada a cabo por John Rawls de su teoría de la justicia bajo el título de liberalismo político, según el cual pueblos y doctrinas razonables pueden estar de acuerdo en el contenido central de los derechos humanos sin compartir las razones para abrazarlos.

Esta solución es, a mi juicio, completamente insatisfactoria. No permite abordar las cuestiones propuestas al principio: la especificidad de la reflexión filosófica sobre los derechos humanos, su relevancia teórica y quizá práctica, y la visión que se tiene desde otras perspectivas teóricas o intereses prácticos de lo que puede ofrecer la filosofía. A mi modo de ver, la filosofía debe abordar su tarea más propia, el análisis conceptual, equipada con la información empírica e histórica relevante y el instrumental analítico necesario. Tanto el preámbulo como el primer artículo de la declaración comienzan afirmando que los seres humanos son libres e iguales en dignidad y derechos. Esa no era entonces, ni es ahora, ni será nunca una proposición empírica. La igualdad de los hombres no es física, sino metafísica. La filosofía ha de analizar la historia y el sentido de esta idea. Se trata de una convención, un sinsentido, una proposición que sólo puede tener sentido religioso... La filosofía es el conjunto de razonamientos y respuestas a cuestiones como ésta, y como la pregunta por la historia y el sentido de las nociones de dignidad y autonomía. Ésta es la tarea propia de la filosofía, una tarea teórica, lo cual no prejuzga que quien haya de ofrecer los análisis y las consideraciones más profundas en cada momento se llame filósofo, físico, jurista o diplomático. Si un diplomático presenta los análisis filosóficos más pertinentes, lo hará en tanto que sujeto que filosofa, no en tanto que diplomático.

¿Cuál puede ser la relevancia práctica de las investigaciones filosóficas sobre los derechos humanos? A mi juicio, esta relevancia existe, aunque no se trata de una relevancia directa, sino indirecta. El trabajo contra la injusticia y por la realización progresiva y efectiva de los derechos humanos no parece corresponder a cualquier concepción de estos derechos, sino a convicciones profundas y específicas acerca de la naturaleza de estos derechos, aunque los filósofos no sepan dar cuenta de ellas. La lucha por la justicia y los derechos está llena de amor y valor, pero presupone también la convicción de que los seres humanos son efectivamente iguales en valor, dignidad y derechos, metafisicamente iguales, no físicamente iguales, ni convencionalmente iguales, aunque el activista no sepa articularlo y los filósofos le intenten convencer de lo contrario. Resultaría muy extraño enarbolar la bandera de los derechos o sacrificar la vida en nombre de una dignidad y una igualdad convencionales. 0 dedicar alma y cuerpo a la lucha por la educación o la integridad física de la gente en nombre de un acuerdo contingente entre voluntades contingentes que tuvo lugar no se dónde. La lucha por los derechos de las mujeres, contra el matrimonio infantil o la reclusión femenina presupone, como afirma Hansa Metha, "la fe en los derechos humanos", aunque Mehta tenga cosas más importantes que hacer que intentar comprender esa fe y dar cuenta de sus implicaciones conceptuales. Con lo cual parece que la relevancia práctica de la teoría se convierte en la relevancia teórica de la práctica, pues la práctica pone de manifiesto que hay teorias que no pueden ser correctas...

Por último, ¿cuál es la relevancia que se atribuye al análisis filosófico desde otras perspectivas teóricas? La economía, el derecho o las relaciones internacionales, por muy volcadas 
que se encuentren a veces en problemas que conciernen a la dignidad y los derechos más básicos de los individuos, no necesitan resolver previamente ningún problema filosófico. Sin embargo, el desinterés o la desconfianza hacia la especulación se encuentra aquí acompañado a menudo por una firmeza de convicciones semejante a la que exhibe el trabajo por la justicia y la realización de los derechos. Si el lenguaje de la disciplina lo requiere, se expresa la convicción del derecho de los individuos a tal cosa, o se asegura que los criterios económicos se deben completar con criterios de equidad, como dicen los economistas. A mi modo de ver, la distancia con la que se observan las disertaciones filosóficas desde el activismo y desde otras disciplinas corresponde unas veces a una clara falta de interés por cuestiones teóricas que tienen gran relevancia para las creencias de estos mismos individuos. Pero otras muchas veces se debe a la incapacidad de la filosofía para presentar análisis sensatos a los que se pueda asomar el lector interesado. La distancia y la impaciencia con la que se contemplan desde fuera las propuestas filosóficas, reflejan a menudo, no una falta de interés por los problemas, sino la perplejidad ante las repetidas manifestaciones de confusión y palabrería con las que los filósofos presentan sus profundos análisis.

\section{LA FILOSOFÍA Y LA JUSTICIA GLOBAL}

En la Introducción a uno de los libros colectivos que ha editado sobre justicia global, Thomas Pogge cuenta que pidió financiación a una fundación importante para organizar el congreso en el que se presentaron las contribuciones recogidas en ese libro. Los evaluadores externos de la fundación rechazaron su solicitud alegando que las cuestiones que se iban a examinar en el congreso demandaban un tratamiento interdisciplinar, desde la economía y el derecho, y que era muy dudoso que los filósofos tuvieran algo interesante que decir. En mi opinión, los escritos y las propuestas del propio Pogge son un buen punto de partida para examinar la relevancia de la filosofía en planteamientos sobre la justicia a escala internacional ${ }^{5}$.

Las teorias modernas de la justicia están concebidas para contextos estatales. Siguiendo el planteamiento seminal de John Rawls, el objeto fundamental de la teoría de la justicia es la estructura básica, es decir, el conjunto de normas y procedimientos económicos y jurídicos en los que se desenvuelve la actividad de los ciudadanos de los estados democráticos. De acuerdo con la propuesta de Rawls, la estructura básica de una sociedad es fundamentalmente justa o injusta si respeta o protege las libertades, la igualdad de oportunidades y el tratamiento diferencial o compensatorio, en alguna medida, hacia los más desfavorecidos. Se trata de una teoría de la justicia estatal 0 , utilizando un anglicismo usual, doméstica (domestic justice), seguida de forma generalizada en la filosofía política contemporánea. El estado es justo o injusto según lo sea su estructura básica, es decir, las normas y procedimientos básicos a los que están sometidos sus ciudadanos.

Ahora bien, las leyes de un estado influyen también, y cada vez más, sobre ciudadanos de otros estados. Influyen de manera directa e inmediata sobre los ciudadanos extranjeros que entran en contacto con ese estado mediante las leyes de inmigración y extranjería, derechos y coberturas para los inmigrantes ilegales, etc. Pero influyen también de forma aparentemente indirecta, pero muy poderosa, sobre otros estados y sus ciudadanos tanto mediante medidas propias, como la política de aranceles, subvenciones o ayudas a la exportación, como a través de sus políticas y posiciones en la arena internacional. Cuando se considera que un estado es justo o injusto no sólo por la influencia que ejercen sus normas y regulaciones sobre la vida de sus propios ciudadanos, sino también por el tipo de influencia y efectos que ejercen sobre la vida de ciudadanos de otros estados, entonces se da el tránsito de la perspectiva de la justicia estatal a la perspectiva de la justicia global ${ }^{6}$. Se trata de un cambio importante de perspectiva que se ha ido generalizando en la última década. Dos ámbitos en los que se ve con claridad las implicaciones de los planteamientos de la justicia global son la concepción de las necesidades humanas y algunas normas y medidas internacionales. Voy a considerar la cuestión inicial sobre la relevancia de la filosofía a propósito de estos dos asuntos.

La noción de necesidades humanas, necesidades básicas universales, es indispensable, como complemento de los derechos humanos, para poder realizar razonamientos y comparaciones transculturales, dar sentido a las nociones de bienestar, calidad de vida y desarrollo humano, e incluso para poder criticar la injusticia y la opresión. Sin una noción decidida de necesidades humanas es imposible la crítica fundada de la adaptación de las preferencias y las 
expectativas a las situaciones injustas. La negación de las necesidades, las capacidades o los derechos más básicos es el aliado más fiel del conservadurismo y el mantenimiento del status quo. Y mostrar esto último es una tarea eminentemente filosófica, aunque, curiosamente, no sean siempre los filósofos los que más han sobresalido en esta tarea. En el pensamiento social de los últimos treinta años, dos hitos importantes para estos propósitos son la teoría de las necesidades humanas de Len Doyal e lan Gough y las teorías de las capacidades humanas de Amartya Sen y Martha Nussbaum? ${ }^{7}$.

Las teorías de las necesidades o las capacidades humanas tienen que analizar una paradoja. Como muestran Doyal y Gough, desde el punto de vista teórico hay muchas maneras de negar la existencia de necesidades o derechos básicos, mientras que las políticas sociales y la práctica jurídica dan por supuesta la existencia de esas necesidades humanas básicas, que reclaman su protección con mayor urgencia moral y normativa que otras. La tarea propiamente filosófica consiste en analizar y desentrañar la complejidad de esta paradoja. Aunque Doyal y Gough no se sitúan profesionalmente en la filosofía, llevan a cabo una tarea filosófica y conceptual de la mayor importancia con la crítica de la negación de las necesidades básicas desde el liberalismo, el socialismo, la antropología, la sociología y el culturalismo. Por su parte, Martha Nussbaum Ileva a cabo el análisis filosófico de las concepciones de la diversidad cultural y las acusaciones de dogmatismo y paternalismo que se utilizan para negar la existencia de capacidades y derechos humanos universales. La tarea filosófica consiste en mostrar la compatibilidad o la incompatibilidad de los derechos humanos y la diversidad cultural, utilizando para ello, de nuevo, las herramientas conceptuales y los datos históricos y empíricos relevantes. En su discurso ante la asamblea general de las Naciones Unidas, el 9 de diciembre de 1948, para pedir la aprobación de la declaración de derechos humanos, uno de los padres de ésta, el diplomático y filósofo chino Peng-chun Chang, la presentó como un documento multicultural, que señalaba el mínimo de decencia en el trato a los seres humanos por parte de la sociedad y los poderes públicos, pero abierto a múltiples especificaciones y a la diversidad de culturas y tradiciones ${ }^{8}$.

Por otro lado, desde una perspectiva global es cada vez más importante pensar la naturaleza y los efectos de normas y procedimientos internacionales que tienen gran influencia en la calidad de vida de muchas personas. Uno de los grandes méritos de Thomas Pogge como teórico de la justicia global es insistir claramente en los efectos nocivos para los estados pobres y sus habitantes de algunas reglas y prácticas en las relaciones internacionales o con relevancia supra-estatal. Destacan dos: las reglas del comercio internacional -en particular el proteccionismo agrícola de los países ricos- y los privilegios de los gobiernos para endeudar a sus estados y vender sus recursos naturales, con independencia de la legitimidad o ilegitimidad de su forma de acceder al poder. En el caso del proteccionismo agrícola, hay tres tipos de medidas gravemente dañinas para los paises pobres: los aranceles, las subvenciones y las ayudas a la exportación. Como demuestran también los documentos preparados por organizaciones como Oxfam y los informes de desarrollo humano de las Naciones Unidas y el Banco Mundial, las medidas proteccionistas de grandes potencias como la UE y EEUU son extremadamente perjudiciales para la agricultura y para la economía general de los países pobres. Los informes muestran que los aranceles que los países ricos imponen a los países pobres son de media cuatro veces superiores a los que se imponen los paises ricos entre sí, que las subvenciones son tremendamente regresivas en el interior de estos paises y que vuelven muy difícil la competencia para los países pobres. Además, los países ricos han inundado muchas veces, sin ningún escrúpulo, los mercados de países pobres con productos no sólo subvencionados, sino beneficiarios de generosas ayudas a la exportación, que han arruinado la producción local.

Pues bien, la pregunta es ¿qué puede ofrecer la filosofía sobre estos fenómenos, que son esenciales para completar los puntos de vista económicos sobre los problemas internacionales con consideraciones de justicia? Mi impresión es que la filosofía, tras ofrecer algunas ideas básicas sobre la justicia, tiene muy poco más que decir. La filosofía politica muestra la estructura de los principios y las nociones más generales de justicia y el sentido de transitar de nociones de justicia estatal a justicia global. Ciertamente, el planteamiento filosófico de la justicia global como referida a normas y estructuras generales de funcionamiento internacional, parece útil y significativo. Y también lo es la propuesta de Thomas Pogge de concebir los deberes asociados como deberes negativos, pues la voluntad de no hacer daño, sin necesidad de apelar a la benevolencia, sería suficiente para comprender y combatir la injusticia de muchas normas estructurales de las relaciones y el 
comercio internacional. Las políticas internas de los países ricos disocian por completo los problemas internos sectoriales, como los de sus agricultores y ganaderos 0 su industria automovilistica, de sus discursos y políticas de ayuda al desarrollo. Una clara inteligencia de la noción de justicia global y de los efectos devastadores de algunas reglas internacionales es incompatible con el discurso de los países ricos sobre sí mismos y sobre sus relaciones con los paises pobres, y ha de tener, ciertamente, una influencia cada vez mayor en la presentación pública de estos problemas.

Sin embargo, la complejidad de los factores económicos y políticos es grande y sólo desde un conocimiento profesional experto se puede llegar a establecer un juicio político acerca de las posibilidades y la conveniencia de acción. El discurso filosófico ofrece una conceptualización adecuada de las nociones de justicia, explicita las diferencias entre los criterios económicos y otros criterios, e incluso puede insistir de forma general en la necesidad de abordar las urgencias de los grupos de presión internos sin olvidar la incidencia de las políticas fuera del territorio nacional. La filosofía puede pedir, quizá, que se incorporen estos elementos y esta conciencia al trabajo y el análisis político. Pero es poco lo que la filosofía como tal, en cuanto disciplina abstracta y conceptual, puede ofrecer para sustanciar o dar concreción a ningún tipo de política internacional. No son tareas de competencia de la filosofía.

Cuando la teorización filosófica sobre la justicia global alcanza alguna concreción política, es obra en realidad de filósofos informados sobre economía o relaciones internacionales. Sus propuestas no las hacen en tanto que filósofos, sino en tanto que conocedores, más o menos informados, de otras disciplinas, de igual manera que el análisis filosófico más relevante sobre los fundamentos o la universalidad de los derechos humanos no tiene por qué ofrecerlo un filósofo, sino que podrá ofrecerlo un científico social o un jurista que filosofa. A mi modo de ver, la apuesta por la justicia global y la realización de los derechos humanos debe ser una prioridad de la actividad política y económica, que ha de hacer suyas consideraciones básicas de justicia. La filosofía es la disciplina que examina despacio la complejidad y las implicaciones conceptuales en éste y muchos otros campos, pero el economista o el político ilustrado puede haber recibido estas nociones de la cultura y la ideología ambiental, accesible a todos, sin necesidad de acudir a la filosofía formal. La realización de la justicia y los derechos humanos son empresas políticas, no filosóficas.

El discurso filosófico sobre la justicia global se encuentra a menudo con un escepticismo como el manifestado por los evaluadores de la propuesta de Pogge para organizar un congreso, a la que me he referido más arriba. A mi juicio, este escepticismo obedece a razones diferentes a las que explican la impaciencia de activistas o teóricos de otras disciplinas ante análisis filosóficos sobre problemas propiamente abstractos. Hans Metha se impacienta ante la reflexión sobre los fundamentos de los derechos humanos y no necesita ni le interesa llevarla a cabo. Pero se trata de un terreno en el que la aclaración conceptual sólo puede provenir del ejercicio propiamente filosófico. En cambio, en el caso de la justicia global, la filosofía puede ofrecer consideraciones relevantes sobre la noción de justicia y la universalidad de las necesidades y los derechos humanos, pero muy poco para la realización efectiva de la justicia y los derechos. La teoría de la justicia y los derechos ilustra y enriquece la ideología y el espíritu de los tiempos. Y la politica debe estar alentada por la voluntad de justicia y la búsqueda del bienestar de todos los seres humanos. Pero no son las reflexiones filosóficas las que permiten llevar la justicia a la actividad política y económica.

\section{NOTAS}

1 Véase, por ejemplo, Gregorio PecesBarba, Ángel Llamas Gascón y Carlos Fernández Liesa, eds., Textos Básicos de Derechos Humanos. Con estudios generales y especiales y comentarios a cada texto nacional e internacional. Elcano, Navarra: Aranzadi, 2001; Micheline Ishay, ed., The Human Rights Reader. Major Political Writings, Essays, Speeches, and Documents from the Bible to the Present New York: Routledge, 1997.
Recibido: 2 de enero de 2009

Aceptado: 23 de febrero de 2009 
2 Tomo todos los datos sobre la elaboración de la declaración de un libro excelente y apasionante: Mary Ann Glendon, A World Made New. Eleanor Roosevelt and the Universal Declaration of Human Rights. New York: Random House, 2001.

3 Mary Ann Glendon, "Foundations of Human Rights: the Unfinished Business", American Journal of Jurisprudence, 14 (1999), 1-14, p. 2.

4 Jacques Maritain, "Introducción", en E. H. Carr y otros, Los derechos del hombre. Barcelona: Laia, 1976. Editado originalmente por la Unesco en 1949.

5 Véase, sobre todo, Thomas Pogge, World Poverty and Human Rights: Cosmopolitan Responsibilities and Reforms. Cambridge: Polity, 2002. Edición española en Paidós.

6 Andreas Follesdal and Thomas Pogge, "Introducción", en A. F. and T. P. eds., Real world justice: grounds, principles, human rights, and social institutions. Dordrecht: Springer, 2005, p. 8.
7 Len Doyal e lan Gough, Teoría de las necesidades humanas (1991). Trad. José Antonio Moyano y Alejandro Colás. ICARIA/FUHEM: Barcelona, 1994; Amartya Sen, Desarrollo y libertad (1999). Trad. Esther Rabasco y Luis Toharia. Barcelona: Planeta, 2000; Martha Nussbaum, Las mujeres y el desarrollo humano: el enfoque de las capacidades (2000). Trad. Roberto Bernet. Barcelona: Herder, 2002.

8 Glendon, A World Made New, op. cit., pp. 226, 230. 